\title{
Carbon C 11 Alpha-methyltryptophan
}

National Cancer Institute

\section{Source}

National Cancer Institute. Carbon C 11 Alpha-methyltryptophan. NCI Thesaurus. Code C95887.

A radiopharmaceutical containing an analogue of tryptophan, alpha-methyltryptophan (AMT), labeled with carbon 11 (11C), used to measure serotonin synthesis in the human brain using positron emission tomography (PET). Upon administration and once it crosses the blood-brain barrier and into the cytoplasm of serotonergic neurons, carbon C 11 alpha-methyltryptophan acts as a substrate for the enzyme tryptophan hydroxylase and undergoes conversion to carbon C 11 alpha-methyl-5-hydroxytryptophan, also known as C 11 alpha-methyl-serotonin (AMS). C 11 AMS accumulates in serotonergic nerve terminals in proportion to the synthesis rate of serotonin because C 11 AMS cannot be broken down by the enzyme monoamine oxidase, and thus the synthesis rate of serotonin can be imaged using PET. C 11 AMT is not incorporated into proteins, nor are metabolites released into the blood pool, making C 11 AMT an excellent tracer for serotonin synthesis in vivo. 